\title{
In Defense of Methodological Mechanism: The Case of Apoptosis
}

\author{
Stavros Ioannidis \& Stathis Psillos \\ Dept of Philosophy and History of Science \\ University of Athens, \\ University Campus \\ 15771 Athens, \\ Greece \\ psillos@phs.uoa.gr \\ sioannidis@phs.uoa.gr
}

\begin{abstract}
This paper advances the thesis of methodological mechanism, the claim that to be committed to mechanism is to adopt a certain methodological postulate, i.e. to look for causal pathways for the phenomena of interest. We argue that methodological mechanism incorporates a minimal account of understanding mechanisms, according to which a mechanism just is a causal pathway described in the language of theory. In order to argue for this position we discuss a central example of a biological mechanism, the mechanism of cell death, known as apoptosis. We argue that this example shows that our philosophically deflationary account is sufficient in order to have an illuminating account of mechanisms as the concept is used in biology.
\end{abstract}

\section{Introduction}

It hardly needs emphasizing that talk of mechanisms is widespread within the biomedical sciences. Pathologists talk about "mechanisms of disease" (Lakhani et al 2009) and pharmacologists about the "mechanisms of action" of drugs (Rang et al. 2016). Molecular biologists talk about the "fundamental mechanisms of life" (Alberts et al. 2014, 22), such as DNA replication and protein synthesis; developmental biologists talk about "genetic mechanisms of animal development" (39), "mechanisms for specifying the germ layers" (Wolpert et al. 2002, 89) and "mechanisms of axis determination" (143); there exist "morphogenetic mechanisms" (254) and the "mechanism of programmed cell death" (Slack 2005, 214).

The aim of this paper is to propose a minimal way of understanding mechanisms and to argue that this conception is sufficient in order to have an illuminating account of scientific practice. We will focus our attention on a central example of a biological 
mechanism, the mechanism of cell death, known as apoptosis. In particular, we are going to use this case in order to advance the philosophical position we call 'methodological mechanism': the claim that to be committed to mechanism is to adopt a certain methodological postulate, i.e. to look for causal pathways for the phenomena of interest. Methodological mechanism and the deflationary account of mechanisms that it incorporates differs from recent accounts of mechanisms in that it is ontologically noncommittal; moreover, our argument will unravel some limitations inherent in any attempt to extract metaphysical conclusions from scientific practice.

We begin in section 2 by introducing our minimal account of mechanisms as causal pathways and then in section 3 we outline the thesis of methodological mechanism. Next, in section 4, after a description of the early research on cell death, we examine the mechanisms of apoptosis focusing on the paper by Kerr et al. (1972) where the term 'apoptosis' was first introduced. We also present the subsequent history of research on apoptosis, which led to its identification as a central biological mechanism. In section 5, we examine the distinction between apoptosis and other mechanisms of cell death. In section 6, we defend our truly minimal view of mechanisms in the context of the apoptosis-oncosis distinction and draw some conclusions concerning the nature of biological mechanisms.

\section{From Metaphysics to Practice}

What kinds of commitments does talk of mechanisms imply? In the philosophy of science, the dominant views about mechanisms until the 1980s had been metaphysical. As we have noted in other work (Ioannidis \& Psillos 2018), mechanism has been seen as a view about causation: mechanisms were taken to provide the missing link (Hume's “secret connexion") between the cause and the effect. 'Mechanism', on this approach, is the very causal connection, and has been described in various ways as mark transmission (Salmon 1984), persistence, transference or possession of a conserved quantity (Mackie 1974; Salmon 1997; Dowe 2000).

In the 1990s, the new mechanical philosophy emerged, which is not a view about causation per se, but rather a view about the causal structure of the world.

Since then, three have been the dominant metaphysical accounts of mechanism:

Mechanisms are entities and activities organized such that they are productive of 
regular changes from start or set-up to finish or termination conditions. (Machamer, Darden, \& Craver 2000, 3)

A mechanism for a behavior is a complex system that produces that behavior by the interaction of a number of parts, where the interactions between parts can be characterized by direct, invariant, change-relating generalizations. (Glennan 2002, S344)

A mechanism is a structure performing a function in virtue of its component parts, component operations, and their organization. The orchestrated functioning of the mechanism is responsible for one or more phenomena. (Bechtel \& Abrahamsen $2005,423)$

We have examined these accounts in some detail elsewhere (cf. Psillos 2004; Ioannidis \& Psillos 2017). What's important to stress for the purposes of this paper is that despite the fact that all these accounts are quite far from traditional mechanistic accounts of causation, they still offer what we might call a 'metaphysically inflated account of mechanism'. In spite of their differences in detail, they are all committed to a certain metaphysics of mechanisms, and in particular to a certain "new mechanical ontology", as Glennan has put it in an unpublished manuscript (forthcoming). This "new ontology" of entities, activities, interactions, organisation of parts into wholes and the like creates the further philosophical need-which mechanists try to meet-to explain what they are and how they relate, if at all, with more traditional metaphysical categories.

The key claims of the New Mechanists then, we think, are three:

1. The world consists of mechanisms.

2. A mechanism consists of objects of diverse kinds and sizes structured in such a way that, in virtue of their properties and capacities, they engage in a variety of different kinds of activities and interactions such that a certain behaviour B or a certain phenomenon $\mathrm{P}$ is brought about.

3. To explain a certain behaviour B or a certain phenomenon P in science is to offer the mechanism of it.

So, though the new mechanists do not commit themselves to a certain global metaphysics of mechanisms - they do not, for instance, align with the seventeenth 
century view of mechanism as configurations of matter in motion subject to laws-their project is not much less metaphysical when they try to offer global accounts of what a mechanism is: the entities might be diverse but they are organised into a mechanism in virtue of their powers, capacities or activities and/or in virtue of their being subjected to laws or at least to invariant generalisations. Mechanisms are typically taken to be things in the world, with objective boundaries, with causally interacting parts bringing about a certain phenomenon $\mathrm{P}$ or manifesting a certain behaviour B. Moreover, the blueprint of a mechanistic explanation is decomposition: the behaviour/function of a system is explained by the interactions/activities of its parts.

In an attempt to offer a less metaphysically committed view of mechanisms, Illari \& Williamson (2012), have noted that "a mechanism for a phenomenon consists of entities and activities organized in such a way that they are responsible for the phenomenon" $(2012,120)$. Glennan has recently accepted a similar view and has characterised it Minimal Mechanism: A mechanism for a phenomenon consists of entities (or parts) whose activities and interactions are organised so as to be responsible for the phenomenon. Glennan takes it that an advantage of this minimal account is that mechanisms are everywhere constituting "the causal structure of the world" (forthcoming, Ch. 2).

Though we think both Illari \& Williamson and Glennan move the issue in the right direction, we take it that, as it stands, their 'minimal' account still invites a number of metaphysical questions that are irrelevant to scientific practice. For instance, they invite almost all of the questions noted above as to the status of entities, their difference from activities, the need to introduce both activities and interactions, the role of the organisation in the performance of the function etc. These might be philosophically legitimate questions to ask, but they need not be asked and answered for an understanding of the role of mechanisms in science.

So, we do not want to claim that the general philosophical accounts of mechanisms are ill-motivated. But we do claim that they inflate the concept of mechanism as this is used in science. Take, for instance, the way pathologists talk about the causes and mechanisms of diseases. They distinguish between causal agents (e.g. viruses) that constitute the aetiology of a disease, and pathogenesis, which concerns the mechanisms, i.e. the causal pathway that leads from the causal agent to a disease state (Lakhani et al. 2009). This can be generalised to other uses of 'mechanism' within life 
sciences, but also more broadly: when scientists talk about 'mechanisms' they do so in the context of searching for a process, i.e. a causal pathway the identification of which would explain how a particular phenomenon is brought about. If the causal pathway is identified, there is little further interest in understanding it according to a certain theory of causation, or to characterise it in terms of entities bearing powers or engaging in activities, or being involved in activities and interactions and the like. In the practice of science, the description of the causal pathway in the language of theory is enough for the identification of the mechanism.

\section{Methodological Mechanism}

A very general, then, and truly minimal way to understand what biologists mean by a 'mechanism' in most (if not all) contexts is this: a mechanism is (simply) the way [causal pathway] a result is brought about. 'Mechanism' concerns how causes (described in the language of theories) operate to bring about a certain effect. To identify a mechanism, then, is to identify a specific causal pathway that connects an initial 'cause' (the causal agent) with a specific result. And if there is a cause for a specific effect, there exists a mechanism that accounts for how the cause operates. The scientific task, then, is to identify the causal pathway. Identification of the causal pathway is crucial in order to establish that a causal link exists between a putative causal agent and a result (e.g. a disease state). But moreover, knowing the causal pathway makes interventions possible (and in the case of pathology, treatment).

Our thesis then is that a thin but methodologically important account of mechanism is the following:

(TMM): mechanism = causal pathway To contrast this view with Glennan’s Minimal Mechanism we call it: Truly Minimal Mechanism (TMM).

Part of the inspiration for TMM comes from what J. H. Woodger called "methodological mechanism" (MM). In his (1929) Woodger distinguished between two ways in which a certain notion can be employed: a metaphysical or ontological way and a methodological one. The latter is when a notion is used for the purposes of description "independently of its metaphysical interpretation". In this case, Woodger says, the notion "is employed methodologically, i.e. simply for the purpose of investigation" $(1929,31)$. The advantage of this use is that the notion can be used in a certain practice and cast 
light on it independently of whatever difficulties (and controversies) are raised by the intricate metaphysical debates concerning what its worldly reference is really like. According to Woodger, taking the methodological standpoint amounts to asking "the methodological mechanist what he has to say in support of his contention that the mechanical explanation is the only one which is admissible in science" $(1929,231)$. Hence, methodological mechanism is a view about mechanistic explanation and its admissibility, and not about the blueprint of the universe. It's not about the metaphysics of mechanism but about the use of the concept of mechanism in science and in particular about the importance of identifying causal pathways. In adopting this view, Woodger noted that mechanism is a "methodological postulate" which as such "makes no assertions about the nature of the processes studied, but merely asserts that they take place according to law, or 'work in an orderly way'” $(1929,258)$.

Taking a cue from Woodger's Methodological Mechanism, we want to claim that commitment to mechanism in science is adopting a methodological postulate which licenses looking for the causal pathways for the phenomena of interest. Hence, MM licenses adopting TMM. TMM (mechanisms=causal pathways) allows for a rich understanding of the use of this concept in biology (and other sciences) without getting embroiled in a debate about what things in the world mechanisms really are and what kind of metaphysical categories their (theory-described) components fall into. Viewing mechanism as a methodological thesis allows that the sought-after identification of the causal pathway by which a specific result is produced, is fully captured in the language of the specific theory, using deeply theory-laden concepts. It forfeits any further need, for the purposes of understanding how mechanisms explain, to offer a further general metaphysical account of how the theory-described entities and processes-the causal pathway-fall into neat metaphysical categories. ${ }^{1}$

It bears stressing that the key feature of MM is that it is non-committal about fundamental ontology. It adopts the postulate that scientists should always try to identify the way that a particular phenomenon is produced, but it says nothing about how causation itself is to be understood (in terms of powers, counterfactuals, etc.), or about what the fundamental ontology of the world is (whether it includes powers, activities, regularities etc.), or even about how the various levels of mechanisms relate to each other (reduction, supervenience etc.). Hence, MM is philosophically neutral. But this does not mean that MM is scientifically neutral. Insofar as it is adopted it licenses 
mechanistic (and only mechanistic) explanations of the phenomena or the behaviours to be explained. Hence, vitalistic explanations are banned, unless 'vital forces' are fed into the causal pathways that produce an effect (a requirement no so easily met, by the way).

Admittedly, TMM is thin. But it does not follow from TMM that mechanisms are not 'things in the world'. After all, they are causal pathways! The point is that there is no need to say something 'deeper' than this in order to have a useful concept of mechanism that elucidates practice: a mechanism (simply) is a sequence of events (or a process) that leads from an initial 'cause' to an end-result. While in pathology and elsewhere there is a distinction between a 'causal agent' and a 'mechanism', strictly speaking when we have a mechanism the cause should be taken not as a single event or an entity, but as a whole sequence of them which lead to the effect. However, we could keep the notion of a 'causal agent' to refer to an event or an entity that initiates a causal pathway, and keep the term 'mechanism' for the causal pathway itself.2

Though MM does not commit us to a specific view about how causation is to be understood from a metaphysical point of view (e.g., it need not commit itself to the view that interaction is the transmission of conserved quantities, etc.), MM can still clarify the close relations between causation, explanation and (the identification of) mechanisms: at least when there is no genuine indeterminism, whatever happens has a prior cause and identifying the way the cause brings about the effect is identifying the causal pathway by means of which the cause operates.

A possible worry here might be that MM (and TMM) are almost vacuous, since everything is (or has) a mechanism: are there things-in-the-world that are not mechanisms? The worry then is that 'mechanism' becomes a concept devoid of real empirical content. Note, by way of reply, that this kind of worry can be effective, if at all, against 'thicker' accounts of mechanism too. It is not clear, for instance, what does not count as a mechanism on Glennan's minimal mechanism account-though on Glennan's earlier views there are non-mechanisms (only) at the level of fundamental physics. Be that as it may, our answer would simply be: some thing is not a mechanism in the TMM sense, if it is not a causal pathway. More importantly, however, the objection has a bite against MM only if MM is taken to be a metaphysical thesis, which is not. As such, the proper contrast is not what-in-the-world-is-not-a-mechanism vs. what-in-the-world-isa-mechanism but rather: are there alternative methodological standpoints? That is, standpoints which explain non-mechanistically? In the 17th century, for example, one 
such alternative methodological standpoint was identified by Boyle as this which explained by reference to Aristotelian substantial forms. In the context of the early twentieth century biology, an alternative methodological standpoint would be vitalism.

Another possible objection to TMM might well be that the mechanism cannot be identified with the causal pathway since the mechanism is an arrangement of entities capable of implementing a causal pathway, whether or not the causal pathway is activated (cf. Illari \& Williamson 2012). In reply, we should note that the causal pathway, as we understand it, and as will be made vivid with the case of apoptosis, does involve entities, since it is entities that are causally connected by the processes of the causal pathway that leads to the required effect. So the mechanism qua the causal pathway obviously involves entities. But to call an arrangement of entities mechanism, in our view, is to make a claim about the causal pathway, which according to the theory does yield a certain effect. In this sense, it is obvious that the causal pathway need not be activated, given the entities; but for the mechanism to cause anything the causal pathway should be initiated.

A final natural worry would be the following: life scientists often talk about organisms or parts of organisms as kinds of machines. But if one regards organisms as machines, isn't one committed to some strong ontological (e.g. reductionist) thesis about organisms? Shouldn't then MM be appropriately modified? The answer is that the machine analogy does not add much to MM. Let us take again a look at the pathology textbook mentioned earlier (Lakhani et al. 2009). In their introduction, the authors stress that they "will adopt a strongly biomedical concept of disease. This is a mechanistic model that regards the body as a machine with repairable or replaceable parts. It looks for specific underlying biological causes and places a high emphasis on the scientific evidence-base for untangling cause and effect in both the disease and its treatment, because this is important for patient care and prognosis" $(2009,4)$. But although they adopt a mechanistic model, "it is a complex model with multiple parts that interconnect. A change in one area is likely to affect another. Thus maintaining homeostasis is not a simple single feedback loop and it is perfectly acceptable that a new equilibrium is achieved under a new set of circumstances, a new baseline; you do not have to return to the original state" $(2009,4)$. On this account, to say that the body is a machine (i.e., to adopt a 'mechanistic model' concerning the body) is to say that the body contains various interacting parts that underlie bodily functions and sometimes 
result in diseases, such that it is possible to identify cause and effect relationships and to intervene to treat parts that malfunction. In other words, the 'mechanistic model' can be seen as a methodological requirement in order to do pathology. Hence, the 'mechanistic model' mentioned by Lakhani et al. is an instance of MM.

One can of course propose stronger requirements for when a system is properly called a 'machine', or a process 'mechanistic'. For example, one can propose a causal modularity criterion, according to which for a process to count as 'mechanistic', there should exist a modular representation of the process (see Woodward 2002). While we could accept such more robust forms of MM, we think that the thesis of MM as outlined here, with its incorporation of TMM (i.e. without commitment to modularity), is sufficient for capturing the many uses of 'mechanism' within the life sciences.

\section{Methodological Mechanism at Work: the Case of Apoptosis}

The best argument in favor of MM and TMM is that this is the conception of mechanism one finds in scientific contexts where the language of mechanisms is used. In order to substantiate this argument from scientific practice, we are going to examine a central example of a type of biological mechanism, i.e. the mechanisms of cell death. This example is particularly instructive for drawing conclusions about the nature of mechanisms in life sciences, since it is a case in which a new mechanism-what came to be known as apoptosis-was identified.

The history of cell death can be traced back to the mid-19th century, when biologists were already aware that there exist processes that lead to the death of cells (cf. Clarke \& Clarke 1996). Cytologists of the time, such as Walther Flemming in 1885, had even observed cells undergoing what we now consider as apoptosis. However, in the following decades and during the 1960s and even later, there was not much interest from biologists in cell death. To explain why this was so, Richard A. Lockshin notes that biologists at that time "tended to think that death was accidental and that mitosis was the active homeostatic process" — cell death was not yet viewed as a "biological process" (Lockshin 2008, 1092).

In the 1960s, new technological developments (e.g. electron microscopy, improved histological techniques) started a new era in the study of cell death. In 1964, Lockshin and Carroll Williams published a paper with the title "Programmed Cell Death" (Lockshin \& Williams 1964). According to Lockshin \& Zakeri, this new expression 
("programmed cell death") was meant to capture the fact that "cells followed a sequence of controlled (and thereby implicitly genetic) steps towards their own destruction" (Lockshin \& Zakeri 2001, 546).

That such a controlled process existed was well known to developmental biologists. John W. Saunders had already noticed that there exist "reproducible patterns of cell death in chick embryos" (Lockshin \& Zakeri 2001, 546) and the same was the case concerning metamorphic cell deaths in insects. As he put it, "abundant death, often cataclysmic in its onslaught, is a part of early development in many animals; it is the usual method of eliminating organs and tissues that are useful only during embryonic or larval life or that are but phylogenetic vestiges" (Saunders 1966,154). Characteristically, Saunders wrote about a "death clock" intrinsic in cells: cells that normally die during chick development, would also die "on schedule" in culture. If, however, they were transplanted to a different area of other chicks, they survived. It was evident from this that cell death is a controlled and regulated process.

To be sure, the idea of "programmed cell death" was metaphorical. As Lockshin explains in a recent review on the history of the subject, it was "a felicitous turn of phrase designed to exploit the trendiness of the then-nascent computer era. The intent was to focus attention on what was relatively obvious: that cell deaths in developing and metamorphosing animals occurred at predictable developmental stages and in specific locations. They must be 'programmed' into the genetics of the organisms, in the same sense that the differentiation and growth of an organ, tissue, structure, or pigment would be considered to be fundamentally determined by the interplay of specific genes" (Lockshin 2016, 10-11).

John F. R. Kerr, who had been working on the processes of cell death since the 1960s, notes that at the time most researchers were "equating cell death with cell degeneration" (Kerr 2002, 472). So, an early hypothesis was that cell death was the result of damaged lysosomes, which were viewed as "suicide bags". Kerr, however, had discovered a certain type of cell death that was "non-degenerative in nature" (472)-he first named it "shrinkage necrosis" (Kerr 1971). This was no accident; the initial thought was that this process was a type of necrosis. But soon Kerr noted that it was a different kind of mechanism-what he and his collaborators called "apoptosis".

Shrinkage necrosis was identified by studying ischaemic liver injury. It was a type of cell death that differed from classical necrosis both morphologically, in that it 
involved scattered cells that were converted in small round bodies, rather than group of cells as in classical necrosis, and also chemically, in that during shrinkage necrosis lysosomes were preserved, again in contrast to classical necrosis were they ruptured. In his 1971 paper, Kerr concluded: "[s]hrinkage necrosis is a distinct and important type of cell death, which has received relatively little attention in the past. It probably results from noxious stimuli that are insufficiently severe to produce coagulative necrosis" (19).

The concept of apoptosis was introduced in a seminal paper in 1972 by Kerr, Andrew H. Wyllie and Alastair R. Currie to refer to what the authors explicitly described as "a hitherto little recognized mechanism of controlled cell deletion" $(1972,239)$. What did Kerr et al. do to identify the mechanism of apoptosis? They described it in the language of theory as a "vital biological phenomenon", which is "complementary to mitosis in the regulation of animal cell populations" (241). This was mainly a description of the specific causal pathway of the deletion of "scattered single cells" (241).

There are two main stages in the apoptotic process as shown by electron microscopy: first, so-called apoptotic bodies are formed; second, apoptotic bodies are phagocytosed and degraded by other cells. Apoptotic bodies are small spherical membrane-bound structures that contain condensed, but otherwise intact and functional, cell organelles and fragments of nuclei. During the formation of apoptotic bodies the nucleus and the cytoplasm condense, the nucleus fragments and protuberances are formed on the surface of the cell. The cell then breaks apart and from the protuberances the apoptotic bodies are formed. Within the cells that phagocytose them, apoptotic bodies show changes that are "very similar to ischaemic coagulative necrosis". But, in contrast to coagulative necrosis, the absence of inflammation in apoptosis results in a process of cell death with minimal disruption of the tissue. So, apoptosis is distinct from necrosis and, as Kerr et al. put it, "is well suited to a role in tissue homoeostasis, since it can result in extensive deletion of cells with little tissue disruption" (250).

Crucially, the morphological changes that occur during apoptosis were "essentially the same" (244) in various types of circumstances studied by the authors, both physiological and pathological. Establishing this point was important, as a mistake that had previously been made in various cases was to confuse apoptotic bodies that have been phagocytosed with, for example, autophagic vacuoles (which often appear 
similar under the electron microscope). For example, apoptosis was found by Kerr and his collaborators to occur spontaneously in both treated and untreated malignant tumors, was involved in cases of pathological atrophy but also of normal involution of tissues, in normal development (e.g. during the development of digits) and in general in cellular turnover in normal adults. It was then taken to be a ubiquitous mechanism: "a distinctive morphological process (...) which plays a complementary but opposite role to mitosis in the regulation of animal cell populations" (255-6).

Incidentally, the description of the mechanism of controlled cell deletion makes clear that the full knowledge of the causal pathway is not necessary for the identification of the mechanism. Kerr et al. noted that "the mechanism" of the all-important condensation of both nucleus and cytoplasm in the first stage of apoptosis was unknown. As they put it, "the condensation is presumably a consequence of the extrusion of water, but its mechanism is still unknown" (244). Moreover, in 1972 little was known "of the factors that initiate apoptosis or of the nature of the cellular mechanisms activated before the appearance of the characteristic morphological changes" (255). Still enough of its causal pathway was known to conclude that "[t]he ultrastructural features of apoptosis and its initiation and inhibition by a variety of environmental stimuli suggest to us that it is an active, controlled process" (256).

By describing the morphological pattern (causal pathway) of the apoptotic process and the various circumstances where it occurs, the authors reach the crucial conclusion that apoptosis has a regulatory role within the organism: it "subserves a general homeostatic function" (Kerr 2002, 471). This regulatory role is the most important difference with coagulative necrosis, the classical type of cell death that had already been described morphologically in detail and which Kerr et al. contrasted to apoptosis. Coagulative necrosis does not regulate cell populations, as it is brought about when homeostatic mechanisms are irreversibly disturbed and is always caused by "noxious stimuli" (Kerr et al. 1972, 239); whereas, apoptosis is caused by both pathological and physiological stimuli. The triggering of apoptosis by physiological stimuli is the reason why apoptosis is considered of great importance by the authors.

Summing up the events that led to the 1972 paper, Kerr writes in a later review that "[a] serendipitous confluence of ideas thus made the formulation of the apoptosis concept virtually inevitable" (Kerr 2002, 473). These ideas involved the realisation that apoptosis is a) a distinctive morphological process, b) ubiquitous and non-disruptive, 
and c) triggered by physiological stimuli. Since then a form of 'physiological cell death' balancing cell populations must exist, and necrosis cannot play this role while apoptosis is well suited to it, apoptosis was proposed as the mechanism with exactly this regulatory role across animals.

By the middle 1970s it was broadly recognised that "cell death was as much a part of cell biology as mitosis, extension of an axon, the enzymatic sequence of glycolysis, or secretion" (Lockshin \& Zakeri 2001, 547). Actually, since its first formulation in 1972, apoptosis has become a fundamental research area in biology. The main reason for this steady increase in importance, especially during the last two decades, was the realisation of its central role in many functions within the organism. By the middle of 1990s, cell death "was recognized as an interesting and biological event"; it was seen not just as "an incidental part of life", but as "a highly controlled and medically important element of existence" (Lockshin \& Zakeri 2001, 545).

In fact, there are three main reasons that can explain why the field of apoptosis was transformed from a modest topic to a central field of biological research. First, it was discovered that apoptosis was much more common than was initially thought by the development of techniques that made it easier to identify instances of apoptosis. Second, conserved genes that control cell death were identified, starting with the genes that determine the developmental pathway for programmed cell death in Caenorhabditis elegans (cf. Ellis \& Horvitz 1986). Unravelling the molecular genetic mechanisms regulating cell death by using Caenorhabditis elegans as a model that began in late 1970s was a breakthrough in the study of regulated cell death. It was thus established that cell death was genetically based, and various important genes that are involved in the regulation of apoptosis were identified (e.g. bcl-2, fas, p53, ced-3, cf. Lockshin \& Zakeri 2001).

The third reason which explains why the field of cell death increased in importance around 1990, were discoveries that showed the clinical relevance of cell death and the relation between it and fields such as immunology and cancer research. For example, the gene bcl-2, a B-cell lymphoma gene, was identified as an anti-apoptotic gene. This established a relation between apoptosis and "differentiation and maintenance of the immune system" (Lockshin \& Zakeri 2001, 549). In the 1990s too, ced-3 in Caenorhabditis elegans was sequenced and it was discovered that it was related to a mammalian protease, and that a family of such proteases exist. Known as caspases, 
these proteases are central components of the apoptosis pathway and their sequence is widely conserved among animals.

The causal pathway of apoptosis can then nowadays be characterised not only morphologically, but also biochemically. We will now briefly describe the main events of the mammalian apoptosis pathway (for a more detailed description see, for example, Shiozaki \& Shi 2004).

Apoptosis can mainly occur via two distinct signalling pathways: the intrinsic pathway, where the apoptotic signal comes from inside the cell, and the extrinsic pathway, where the apoptotic signal comes from outside. Common to both pathways is what is known as the caspase cascade. Caspases are enzymes that perform proteolysis and are central components in both pathways. They exist in the cytoplasm under normal conditions, but not in an active form. For apoptosis to occur, some caspases need to be activated. In turn, these active caspases activate other caspases; this is the caspase cascade, which eventually leads to the morphological changes of apoptosis mentioned earlier.

The extrinsic signalling pathway starts with the binding of an extracellular death ligand to a death receptor. For example, T-lymphocytes have a Fas ligand which can bind to the Fas receptor, a protein located on the surface of the cell. The binding of the Fas ligand to the Fas receptor is the signal for the cell to commit suicide. The Fas receptor has a domain within the cell (FADD -Fas Associated Death Domain); when the ligand binds to the Fas receptor, FADD is activated and recruits the FADD adaptor protein. Subsequently, procaspase- 8 or 10 binds to the adaptor protein. The formation of this complex (known as DISC -Death Inducing Signalling Complex) is the signal for the caspase cascade to occur. The procaspases are cleaved and active caspases 8 or 10 are formed. These initiator caspases activate the effector caspase-3 that breaks down intracellular proteins.

Central component in the regulation of the intrinsic signalling pathway is the bcl-2 family of proteins. This pathway is initiated, for example, when there is DNA damage that cannot be repaired. A central event in this pathway is the release of cytochrome $c$, a protein that normally exists in the intermembrane space of mitochondria, into the cytosol. The release of cytochrome $c$ initiates the caspase cascade. This release occurs when pro-apoptotic bcl-2 proteins (Bax, Bak) aggregate to form a channel in the outer mitochondrial membrane. In normal circumstances there is 
a balance between anti-apoptotic and pro-apoptotic proteins: anti-apoptotic proteins (like Bcl-2 and Bcl-xL) bind to the pro-apoptotic ones, thereby stopping them from forming the channels. But when DNA is damaged, other pro-apoptotic bcl-2 proteins (BH3-only proteins) are synthesised, which bind to the anti-apoptotic ones, thereby inhibiting the inhibitors. When cytochrome $c$ is released into the cytosol, it binds to Apaf-1 (Apoptotic protease activation factor 1). This in turn causes Apaf-1 proteins to oligomerise and form the apoptosome, which activates caspase-9 (the initiator caspase), which in turn activates the effector caspase-3 that breaks down intracellular proteins.

\section{Mechanisms of Cell Death}

An important issue that crops up here for MM is how the various causal pathways are identified and distinguished from each other. Could it be the case that some causal pathways are mechanisms in a more robust sense while others are merely causal pathways? In the case at hand, the issue is how apoptosis can be distinguished from other cell-death processes. As we have noted already, the activation of caspases underlies the morphological changes that occur during apoptosis. However, not all processes of cell death are apoptotic (i.e. not all processes that lead to cell death feature the particular sequence of morphological changes associated with apoptosis), neither do all involve caspase activation. This is why in the literature on cell death there are more general terms that are used to refer to the various types of processes of cell death: on the one hand, there is 'physiological' or 'regulated' cell death; on the other, 'accidental cell death'. Lockshin \& Zakeri explain the difference between the two by noting that when cells die as a result of a process of physiological cell death, "such deaths are part of the normal function of the organism"; also, in physiological cell death genetic regulation is central. This is not the case in "necrosis or oncosis which is accidental and in which the cell has no active role" (Lockshin \& Zakeri 2001, 545).

Could it then be the case that there is a genuine difference between apoptosis and necrosis such that apoptosis is a mechanism in a more robust sense than just the causal pathway for cell deletion, whereas necrosis is merely a causal pathway? To address this issue, let us note first that as Majno \& Joris (1995) have pointed out, apoptosis and necrosis should not be juxtaposed: apoptosis is a process that leads to cell death, but necrosis should not be used to refer to such a process. As they stress, there is a distinction between cell death and necrosis: cell death comes about before necrotic 
changes can be observed. Necrotic changes (for example, karyolysis, karyorhexis, loss of structure in the cytoplasm) "are the features of a cell's cadaver, whatever the mechanism of the cell's death, be it ischaemia, heat, toxins, mechanical trauma, or even apoptosis" (Majno \& Joris 1995, 11). This has actually been the traditional meaning of necrosis, i.e. it refers to changes in tissues that are visible even without a microscope and as such occur after cell death. 3

This point has been emphasised also by other authors. So, according to Kanduc et al. $(2002,167)$, it is "scientifically unjustified" and "unsound" to compare apoptosis to necrosis, as apoptosis is a process that leads to cell death, whereas necrosis refers to changes that occur to cells after they die. In general then, we should distinguish between the processes that a dying cell undergoes (e.g. apoptosis) and the end result of these processes, which is the dead cell. 'Necrosis' should then refer to already dead cells and tissues and the changes that occur after cell death (see also Fink \& Cookson 2005). This usage of the term 'necrosis' is precisely the one suggested by the Committee on the Nomenclature of Cell Death chartered by the Society of Toxicologic Pathologists to make recommendations "about the use of the terms 'apoptosis' and 'necrosis' in toxicity studies" (Levin et al. 1999, 484). In line with the above, this Committee recommended that "when dead cells or tissues are observed in a histological lesion, 'necrosis' is the appropriate morphological diagnosis, regardless of the pathway by which the cells or tissues died" (Levin et al. 1999). They conclude: "[t]his Committee believes that returning to the long-established histopathological standard wherein the word necrosis denotes dead cells in a living tissue (regardless of their phenotype) should help to alleviate the confusion attendant on the notion, held by many, that a dichotomy exists between apoptosis and necrosis" (Levin et al. 1999). Notably, Sloviter $(2002,22)$ goes as far as to note that "the term necrosis is now virtually meaningless because 'necrotic' means nothing more than 'dead'”.

According to Majno and Joris, "the major sore spot in the nomenclature of cell death is precisely the lack of a suitable name for cell death that occurs not by apoptosis but by some external agent" (Majno \& Joris 1995, 11). And this is precisely the point: cell death might have different causal pathways and the difference between them is not that one (or some) of them counts as a mechanism while the other does not; rather the difference is in how they are described.

Majno and Joris's own suggestion is to characterise apoptosis in contrast with a 
specific process of cell death they call "oncosis" $(1995,12)$. A common non-apoptotic causal pathway that leads to cell death is when groups of cells die of ischaemia (ischaemic necrosis). During the causal pathway that leads to ischaemic necrosis the cell swells and it is in order to capture this swelling process that the authors propose 'oncosis' as a term to refer to this mechanism. This causal pathway can nowadays be characterised in detail: reduced supply of oxygen and nutrients leads to ATP depletion, which ultimately results in protein denaturation, enzymatic digestion due to damaged lysosomes and loss of integrity of the plasma membrane, resulting in influx of water and calcium into the cell, leading to swelling and ultimately rupture of the cell. Also, we know that ischaemia typically activates the causal pathway, but toxic agents can also initiate it. We can then talk about the mechanism of oncosis in our minimal methodological sense of the term. Oncosis and apoptosis, then, are two causal pathways (and hence mechanisms) of cell death -by swelling and by shrinkage, respectively.

However, there might be a way to distinguish apoptosis from oncosis such that only the former counts as a mechanism. We will consider three distinctions used by researchers of cell death that might be used in order to do this: i) processes of physiological vs. accidental cell death, ii) processes of programmed vs. non-programmed cell death and iii) active vs. passive processes.

A term widely used to describe a non-apoptotic, non-physiological type of cell death is 'accidental cell death'. By describing a process of cell death as 'accidental', biologists try to capture the idea that, in contrast to apoptosis, this is not a process that occurs under normal conditions, nor does it serve a general homeostatic function within the organism. However, as Majno and Joris (1995) note, apoptosis can also be induced by a variety of 'accidental' causes (e.g. heat, chemical agents, viruses). Levin et al. also note that "dead cells having the cytological features of apoptosis can occur in large numbers as a pathological change, e.g., 'single cell necrosis' in the liver and lymphocyte necrosis in the thymus, and that these changes can be induced by exogenous events such as exposure to toxicants" (Levin et al. 1999, 485). So, the very fact that apoptosis can be initiated by 'accidental causes' shows that the right contrast here is not between mechanism and non-mechanism, but rather between physiological cell death and accidental cell death, where 'physiological cell death' refers to a process of cell death that was initiated by physiological stimuli. 
What about the programmed - non-programmed distinction? An important point here is that 'programmed cell death' and apoptosis should not be identified; thus, the former cannot be used to distinguish the latter. Programmed cell death is the phenomenon where cells die 'on schedule', i.e. they are programmed to die at a specific time. During development of the chick, for example, the morphology of the wing is produced as a result of the death of groups of cells; there is a 'genetic clock' that determines when the cells will die. But when the time comes for the cell to die, the specific programme that determines the form that cell suicide will take, is triggered. The particular form of cell suicide can be apoptosis (indeed, very frequently it is), but it need not be. As Majno and Joris stress: "The genetic programme of programmed cell death is a clock specifying the time for suicide, whereas the genetic programme of apoptosis specifies the weapons (the means) to produce instant suicide" $(1995,11)$ : the weapon is precisely what we call the causal pathway. Again, the point here is that one cannot use this distinction to distinguish apoptosis as a genuine mechanism.

Biologists, we noted above, have characterised the contrast between processes of cell death along the active-passive lines: apoptosis was described as 'active' from the very beginning of its introduction, and contrasted with the 'passive' necrosis 4 . Perhaps what makes apoptosis a mechanism, then, is precisely that it is an active process. However, what does this distinction really mean? The idea here seems to be that in the case of apoptosis the cell is itself involved in its own demise ('cell suicide'), whereas in the case of oncosis the cell dies as a result of some exogenous influence ('cell murder'). That is, apoptosis involves a 'suicide programme' that is initiated under various circumstances and that is genetically based, in the sense that there exist specific genes that code for various components of the biochemical pathway underlying the apoptotic process (the distinctions regulated vs. non-regulated, ordered vs. unordered, controlled vs. non-controlled appear to be used in a similar way).

To make this clear, Sloviter $(2002,23)$, after describing the two causal pathways as 'active cell death' (ACD) and 'passive cell death', notes that ACD is active in the sense that it requires "active intracellular processes for death to result", whereas in passive cell death "the cell plays no role in its own demise", that is, cell death is "immediate and involves no cellular activity", the cause being exogenous to the cell such as "rapid freezing, aldehyde fixation, heat denaturation, and catastrophic physical destruction". As such, passive cell death is of little interest since being immediate it "offers no 
therapeutic window".

The important point for us is that this difference between 'active' and 'passive' is merely a difference concerning the details of each causal pathway. Hence, there is no intrinsic difference between the two causal pathways as such: there is nothing particularly active in apoptosis and particularly passive in oncosis. The significant difference from a biological point of view is that because apoptosis involves a 'suicide programme', it can serve a homeostatic function, as argued by Kerr et al. (1972).

To avoid the insinuation that the use of 'active' and 'passive' processes might lead to views about the ontology of causation and mechanisms (e.g. to the distinction between entities and activities, as in MDC (2000)), let us see how biologists view the active-passive distinction. Kanduc et al. say: "[i]t is frequently assumed that the death of cells can be passive. This non-biological point of view on cell death ignores the role of cell death in cell development and adaptation. It cannot be assumed that 'ordinary' cell death or 'necrosis' is a passive process while the presumed special form of cell death, 'apoptosis' is active. Both the ante-mortem and postmortem changes are active since both are enzyme-catalysed biochemical reactions" (Kanduc et al. 2002, 167-168, emphasis added).

\section{Is Mechanism more than the Causal Pathway?}

Hence, apoptosis and oncosis can both be considered mechanisms in the truly minimal sense: they are both causal pathways that produce a result (apoptotic and ischaemic necrosis, respectively). However, the history of programmed cell death and apoptosis during the last 60 years might be used to argue that in biological practice what counts as a biological mechanism cannot just be a matter of identifying a specific causal pathway. Apoptosis seems to be a special kind of causal process with distinctive features that deserves to be labeled a mechanism. This can even been seen in biologists' description of apoptosis as a 'mechanism of cell death'.

Note that the reason apoptosis became a central biological mechanism is not due to some feature internal to the sequence of events that constitutes the apoptotic pathway, but rather due to features that are external to the pathway itself, i.e. because it is a key process that controls homeostasis. It is its role within the developing and adult organism that led to the formulation of the concept by Kerr et al. in their (1972); similarly, it is the discovery of its highly controlled nature and conservation of the 
genetic sequences of the components of the apoptotic pathway across animals, as well as the realisation of the close relation between apoptosis and the immune system and cancer that followed the molecular genetic discoveries of the 1980s and 1990s, that gave it the central prominence it deservedly has today as a biological phenomenon.

To put the point differently, what we think the story of apoptosis shows, is the following: in the world there are causal pathways for various phenomena; all causal pathways can be deemed mechanisms in our truly minimal sense; but not all those causal pathways are biologically interesting or significant, even if they occur frequently within organisms. Biologically interesting or significant causal pathways are those pathways that subserve a central function within the organism; that is, whether a causal pathway is biologically interesting has to do with features external to the pathway itself.

This does not imply that what is biologically interesting is something subjective. Rather, it implies that it is directly related to biological practice: what the community of biologists regards as the basic phenomena that must be explained in order to have both biological understanding and apply our knowledge clinically. Thus we could say that what makes a causal pathway a specifically biological mechanism is not something internal to the pathway itself; rather, it concerns the role of that pathway within the organism. In other words, whether a causal pathway is considered a biological mechanism by biologists, has to do with a relational property of the pathway. In the case of apoptosis, this relational property is the homeostatic function that it subserves. This relational property is the difference between a causal pathway like apoptosis and a causal pathway such as oncosis.

However, suppose one were to argue as follows. We should certainly let biological practice itself decide what we should mean by a 'mechanism' in a biological context. If practice has it that a causal pathway is deemed a mechanism by an appeal to external features of the pathway, so be it. TMM (the point would be) is false, since

(P-TMM): mechanism=causal pathway $+\mathrm{X}$, where $\mathrm{X}$ is some biologically significant external feature of the causal pathway.

Now, if one were to argue like this, we would not seriously object. We are ready to accept that there may well exist features external to a particular causal pathway, and in particular features that can be established by looking at biological practice, which 
determine whether a specific theory-described causal pathway counts as a biological mechanism. But, in our view, this attribution of 'mechanism' to certain causal pathways and perhaps not to others would entail that 'mechanism' is an honorific term attached to causal pathways that have certain (external) features. The further scientific question then is whether there is evidence that a causal pathway is a 'mechanism' in this sense or not.

In our view, the choice between TMM and P-TMM is not particularly significant: to adopt P-TMM is to claim that we allow a distinction between a causal pathway for a phenomenon $\mathrm{P}$ and a specifically biological mechanism, where the difference between the two concerns an external feature of the respective causal pathways. Be that as it may, the important point is that both views are licensed by methodological mechanism: to be committed to either option, one need not be committed to some metaphysical view about causation or the ontology of mechanisms. There is no need to do this in order to understand scientific practice.

Could someone insist that there is some other feature that differentiates a causal pathway from a mechanism? A possibility here is to adopt the requirement of causal modularity (cf. Woodward 2002). Causal modularity may be seen as the criterion that determines whether a process counts as machine-like or not; so, perhaps 'mechanism' should be used only for causal processes that exhibit causal modularity. While modularity can be important in many cases as a requirement for a causal representation of a system (this is the robust form of methodological mechanism we mentioned in the beginning), the major disadvantage of this view is that many instances of 'mechanisms' in biology turn out not to be such, since they are not modular; apoptosis is a case in point (cf. Cassini 2016). So, adopting this view necessitates abandoning taking scientists' talk of mechanisms at face value.

Lastly, consider again the worry mentioned in section 3, according to which not every mechanism is a causal pathway, since there may exist 'inactivated' mechanisms. Suppose examples of cases of 'mechanisms without activities' are cases of 'mechanisms' that instead of producing a change maintain stability (cf. Illari \& Williamson 2012). It should then be clear that homeostatic mechanisms within cells that prevent apoptosis do involve causal pathways (e.g. the causal pathway in which anti-apoptotic proteins bind to pro-apoptotic ones). So, a causal pathway need not result in a specific change; its end-result may well be the maintaining of a stable state. 


\section{Conclusions}

Most philosophical accounts of mechanisms offer 'inflated' accounts of what mechanisms are, raising questions about the ontology of mechanisms that seem not necessary in order to understand scientific practice. In contrast to such accounts, in this paper we have defended a deflationary account of mechanisms: according to what we called Truly Minimal Mechanism, a mechanism just is a causal pathway that produces a particular effect. Moreover, we claimed that commitment to mechanism in science means adopting a certain methodological postulate, i.e. that one should always look for the causal pathways producing the phenomena of interest. As such, it does not make any general ontological assertions about the nature of causal processes.

We used the example of apoptosis to show that several distinctions used by biologists in order to differentiate between causal pathways and identify the genuine biological mechanisms (active vs. passive, programmed vs. non-programmed, physiological vs. accidental) do not correspond to internal features of causal pathways, but concern an external feature, i.e. the role those processes play within the organism.

What the case of apoptosis shows, then, is that a truly minimal understanding of mechanisms is all that is needed in order to understand biological practice.

Acknowledgments: We wish to thank the audience of the 'Mechanisms in Medicine' workshop (Centre of Reasoning, University of Kent, UK), where a previous version of this paper was presented, for their valuable feedback.

\section{References}

Alberts, B., Johnson, A., Lewis, J., Raff, M., Roberts, K. and Walters, P. (2014) Molecular Biology of the Cell (6th ed). Garland Science

Bechtel, W. and A. Abrahamsen (2005) Explanation: A Mechanistic Alternative. Studies in History and Philosophy of Biological and Biomedical Sciences 36: 421-441

Brandon, R. (1990) Adaptation and Environment. Princeton University Press

Casini, L. (2016) Can Interventions Rescue Glennan's Mechanistic Account of Causality?. British Journal for the Philosophy of Science 67: 1155-1183

Clarke, P. G. H. and S. Clarke (1996) Nineteenth Century Research on Naturally Occurring Cell Death and Related Phenomena. Anatomy and Embryology 193: 81-99

Dowe, P. (2000) Physical Causation. Cambridge: Cambridge University Press 
Ellis, H. M. and H. R. Horvitz (1986) Genetic Control of Programmed Cell Death in the Nematode C. elegans. Cell 44: 817-829

Fink, S. L. and B. T. Cookson (2005) Apoptosis, Pyroptosis, and Necrosis: Mechanistic Description of Dead and Dying Eukaryotic Cells. Infection and immunity 73: 1907-1916

Glennan, S. (2002) Rethinking Mechanistic Explanation. Philosophy of Science, 69: S342-S353

Glennan, S. (forthcoming) The New Mechanical Philosophy. Oxford: Oxford University Press

Illari, P. M. and J. Williamson (2012) What is a Mechanism? Thinking about Mechanisms across the Sciences. European Journal of Philosophy of Science 2: 119-135

Ioannidis S. and S. Psillos (2017) Mechanisms, Counterfactuals and Laws. In: Glennan S. and P. M. Illari (eds) The Routledge Handbook of Mechanisms and Mechanical Philosophy. New York: Routledge, pp.144-156

Kanduc D., A. Mittelman, R. Serpico, E. Sinigaglia, A. A. Sinha, C. Natale, R. Santacroce, M. G. Di Corcia, A. Lucchese, L. Dini, P. Pani, S. Santacroce, S. Simone, R. Bucci and E. Farber (2002) Cell Death: Apoptosis versus Necrosis. International Journal of Oncology 21: $165-170$

Kerr, J. F. R. (1971) Shrinkage Necrosis: a Distinct Mode of Cellular Death. Journal of Pathology 105: 13-20

Kerr, J. F. R. (2002) History of the Events Leading to the Formulation of the Apoptosis Concept. Toxicology 181-182: 471-74

Kerr, J. F. R., A. H. Wyllie and A. R. Currie (1972) Apoptosis: A Basic Biological Phenomenon with Wide-Ranging Implications in Tissue Kinetics. British Journal of Cancer 26: 239-57

Lakhani et al, S., Dilly, S., Finlayson, C. (2009) Basic Pathology: An Introduction to the Mechanisms of Disease (4th ed). Hodder Arnold

Levin S., T. J. Bucci, S. M. Cohen, A. S. Fix, J. F. Hardisty, E. K. LeGrand, R. R. Maronpot, B. F. Trump (1999) The Nomenclature of Cell Death: Recommendations of an ad hoc Committee of the Society of Toxicologic Pathologists. Toxicologic Pathology 27: 484-490

Lockshin, R. A. (2008) Early work on apoptosis, an interview with Richard Lockshin. Cell Death and Differentiation 15: 1091-1095

Lockshin, R. A. (2016) Programmed Cell Death 50 (and beyond). Cell Death and Differentiation 23: 10-17

Lockshin, R. A. and C. M. Williams (1964) Programmed Cell Death-II. Endocrine Potentiation of the Breakdown of the Intersegmental Muscles of Silkmoths. Journal of 
Insect Physiology 10: 643-9

Lockshin, R. A. and Z. Zakeri (2001) Programmed Cell Death and Apoptosis: Origins of the Theory. Nature Reviews Molecular Cell Biology 2: 545-50

Machamer, P., L. Darden and C. F. Craver (2000) Thinking About Mechanisms.

Philosophy of Science 67: 1-25

Mackie, J. L. (1974) The Cement of the Universe. Oxford: Clarendon Press

Majno G. and I. Joris (1995) Apoptosis, Oncosis, and Necrosis. An Overview of Cell Death. American Journal of Pathology 146: 3-15

Oulis, P. (2010) Nature and Main Kinds of Psychopathological Mechanisms. Dialogues in Philosophy, Mental and Neuro Sciences 3: 27-34

Proskuryakov, S. Y. and Gabai, V. L. (2010) Mechanisms of Tumor Cell Necrosis. Current Pharmaceutical Design 16: 56-68

Psillos, S. (2004) A Glimpse of the Secret Connexion: Harmonising Mechanisms with Counterfactuals. Perspectives on Science 12: 288-319

Rang, H. P., J. M. Ritter, R. J. Flower and G. Henderson (2016) Rang \& Dale's Pharmacology (8th ed). Churchill Livingstone

Salmon, W. (1984) Scientific Explanation and the Causal Structure of the World. Princeton, NJ: Princeton University Press

Salmon, W. (1997) Causality and Explanation: A Reply to Two Critiques. Philosophy of Science 64: 461-77

Saunders, J. W. Jr. (1966) Death in Embryonic Systems. Science 154: 604-612

Shiozaki, E. N. and Y. Shi (2004) Caspases, IAPs and Smac/DIABLO: mechanisms from structural biology. Trends in Biochemical Sciences 39: 486-494

Slack, J. M. W. (2005) Essential Developmental Biology (3rd ed). Wiley-Blackwell

Sloviter, R. S. (2002) Apoptosis: a Guide for the Perplexed. Trends in Pharmacological Sciences 23: 19-24

Wolpert, L., R. Beddington, T. Jessell, P. Lawrence, E. Meyerowitz and J. Smith (2002) Principles of Development (2nd ed). Oxford: Oxford University Press

Woodger, J. H. (1929) Biological Principles: A Critical Study. London: Routledge \& Kegan Paul Ltd

Woodward, J. (2002) What is A Mechanism? A Counterfactual Account. Philosophy of Science 69: S366-S377 
1 For a view along similar lines, consider this quotation from Brandon (1990, 185, emphasis added): “A causal/mechanical explanation is one that explains the phenomenon of interest in terms of the mechanisms that produced the phenomenon. What is a mechanism? ... [T] his question has no general metaphysical answer, because the business of science is the discovery of mechanisms; so we cannot delimit in any a priori manner the mechanisms of nature. ... The best we can do is to give an open-ended answer: a mechanism is any describable causal process".

2 Let us also note here in passing that in the recent literature on mechanisms, mechanisms are very often regarded as specific kinds of systems. We think that this is misleading, since mechanisms and systems are different things: mechanisms on our view are causal pathways, and it is strange to call a causal pathway a 'system' (see also Oulis 2010).

3 For example, here is how Virchow in the 19th century describes necrosis in Cellular Pathology: "In necrosis we conceive the mortified [gangrenous] part to be preserved more or less in its external form" (quoted in Majno \& Joris 1995, 3-4).

${ }^{4}$ However, see Proskuryakov \& Gabai (2010) for the argument that necrosis can in certain cases be considered as an active and well-regulated process. 